\title{
Testing telediagnostic thyroid ultrasound in Peru: a new horizon in expanding access to imaging in rural and underserved areas
}

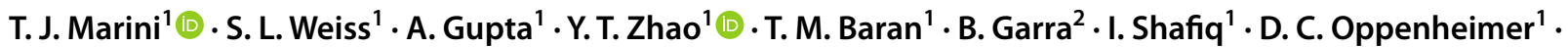

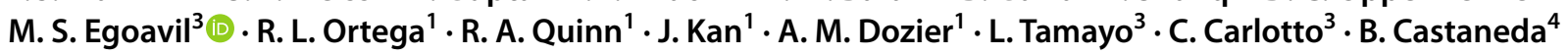

Received: 11 February 2021 / Accepted: 21 April 2021 / Published online: 10 May 2021

(c) The Author(s) 2021

\begin{abstract}
Purpose Thyroid ultrasound is a key tool in the evaluation of the thyroid, but billions of people around the world lack access to ultrasound imaging. In this study, we tested an asynchronous telediagnostic ultrasound system operated by individuals without prior ultrasound training which may be used to effectively evaluate the thyroid and improve access to imaging worldwide. Methods The telediagnostic system in this study utilizes volume sweep imaging (VSI), an imaging technique in which the operator scans the target region with simple sweeps of the ultrasound probe based on external body landmarks. Sweeps are recorded and saved as video clips for later interpretation by an expert. Two operators without prior ultrasound experience underwent $8 \mathrm{~h}$ of training on the thyroid VSI protocol and the operation of the telemedicine platform. After training, the operators scanned patients at a health center in Lima. Telediagnostic examinations were sent to the United States for remote interpretation. Standard of care thyroid ultrasound was performed by an experienced radiologist at the time of VSI examination to serve as a reference standard.

Results Novice operators scanned 121 subjects with the thyroid VSI protocol. Of these exams, $88 \%$ were rated of excellent image quality showing complete or near complete thyroid visualization. There was $98.3 \%$ agreement on thyroid nodule presence between VSI teleultrasound and standard of care ultrasound (Cohen's kappa 0.91, $P<0.0001$ ). VSI measured the thyroid size, on average, within $5 \mathrm{~mm}$ compared to standard of care. Readers of VSI were also able to effectively characterize thyroid nodules, and there was no significant difference in measurement of thyroid nodule size $(P=0.74)$ between VSI and standard of care.

Conclusion Thyroid VSI telediagnostic ultrasound demonstrated both excellent visualization of the thyroid gland and agreement with standard of care thyroid ultrasound for nodules and thyroid size evaluation. This system could be deployed for evaluation of palpable thyroid abnormalities, nodule follow-up, and epidemiological studies to promote global health and improve the availability of diagnostic imaging in underserved communities.
\end{abstract}

Keywords Global health · Goiter · Telemedicine $\cdot$ Thyroid cancer $\cdot$ Thyroid ultrasound · Ultrasound

\section{Introduction}

B. Castaneda

castaneda.b@pucp.edu.pe

1 University of Rochester Medical Center, 601 Elmwood Ave, Box 648, Rochester, NY 14642, USA

2 Medical Imaging Ministries of the Americas, 10810 Lake Minneola Shores, Clermont, FL 34711, USA

3 Medical Innovation and Technology, Calle Los Libertadores 635, 15046 San Isidro, Peru

4 Pontifica Universidad Catolica del Peru, Av. Universitaria 1801, 15088 San Miguel, Peru
Thyroid disease is ubiquitous throughout the world affecting millions of people [1]. Thyroid ultrasound is an established pillar in the diagnosis and management of palpable thyroid findings and thyroid cancer, but at least $2 / 3$ of the world lacks access to ultrasound imaging [2-5]. While ultrasound is a cost-effective diagnostic modality, the lack of trained sonographers who require costly and time-consuming training on internal anatomy and ultrasound operation limits its deployment [6 78]. Furthermore, limitations in healthcare infrastructure including a lack of trained personnel and high-speed internet connections stymie many telediagnostic 
approaches which are otherwise viable solutions to bring imaging to underserved areas [9-12].

To address these problems, a new telediagnostic system utilizing ultrasound volume sweep imaging (VSI) has been previously piloted (Fig. 1) [13]. In this system, images are obtained by individuals without prior ultrasound experience after a few hours of training on ultrasound VSI protocols which are based on simple external body landmarks. In VSI, the operator sweeps the probe over the target area to acquire cine clips which are then sent for remote interpretation. In this asynchronous system, clips can be acquired without internet and sent over low internet bandwidths [14]. In contrast, real-time telemedicine systems require higher bandwidths for videoconferencing and the concurrent presence of a specialist which are often not available in rural and underserved areas $[9,15,16]$.

Using teleultrasound to detect thyroid nodules and evaluate palpable findings could be a great asset in improving the health of the global community. Goiters remain prevalent in rural and underserved areas throughout the world; in one sample of children in northwest Ethiopia, there was a goiter prevalence of $37.6 \%$ [17-20]. If large enough, a goiter can compress the airway, and thyroid ultrasound can accurately assess thyroid size [21-23]. Goiters and palpable abnormalities also require evaluation for malignancy as studies have shown up to a $20 \%$ concurrent incidence of cancer in goiter $[24,25]$. There is an increasing incidence of thyroid cancer with a global distribution including in South America where this study was conducted [26-33]. Thyroid ultrasound is a first-line examination for thyroid nodules and is used to follow and evaluate them as standard of care [34, 35]. In many areas of the world, including South America, there is a lack of information regarding thyroid nodules/cancer incidence $[33,36]$. Thyroid teleultrasound could also be an important epidemiological tool to close this knowledge gap.

Our previous pilot of the teleultrasound system found that the thyroid gland was well evaluated with VSI thyroid ultrasound [13]. In the vast majority of pilot examinations, the

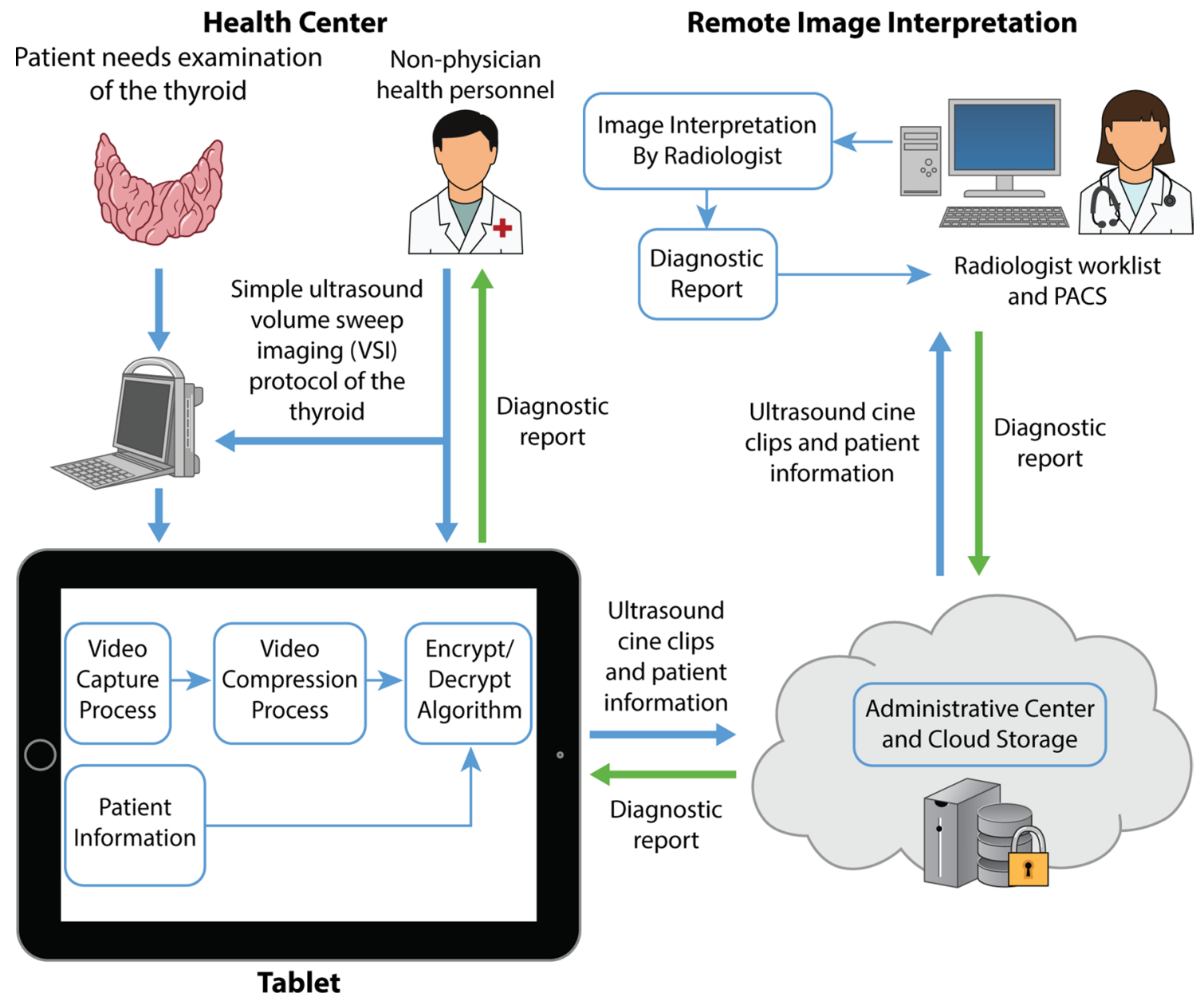

Fig. 1 Thyroid teleultrasound. This figure shows a schematic overview of the thyroid teleultrasound system. Images acquired by individuals without prior ultrasound training are sent for remote inter-

pretation via the use of a tablet. The imaging report is subsequently returned back to the tablet for review by the ordering clinician after interpretation by a radiologist 
entire thyroid gland was visualized with acceptable imaging quality. However, a relatively small sample $(n=22)$ and the lack of a ground truth for comparison were major limitations. In this study, we aimed to test the thyroid VSI protocol in a larger sample with an available ground truth reference standard. Based on the pilot data, we hypothesized that the thyroid would be completely visualized in the majority of examinations with interpretations in agreement with standard of care imaging.

\section{Materials and methods}

\section{Thyroid VSI}

VSI is an imaging technique designed to increase access to imaging in underserved and rural areas [37]. In VSI, the operator requires no significant anatomic knowledge or technical skill to acquire the imaging. The basic component of any VSI protocol is a sweep of the ultrasound probe over predetermined, easily recognized external body landmarks. For example, in the thyroid VSI protocol, the first sweep involves placing the probe under the chin and sweeping to the base of the neck. The cine clip of the sweep is recorded and sent for remote interpretation. In this manner, the novice operator is not responsible for any imaging interpretation. Presets of the ultrasound machine are used which further obviate the need for technical skill or adjustments of the ultrasound machine. The probe is swept at a rate of $1-2 \mathrm{~cm} / \mathrm{s}$ and produces an image every 1-2 mm [13]. Additional arcing (fanning) of the probe at the beginning and at the end of each sweep maximizes visibility. When combined, all acquired cine clips comprise a full volumetric acquisition of the target organ system.

Thyroid, obstetric, right upper quadrant, and lung VSI protocols have all been tested with encouraging results $[13,38-40]$. Training data from these studies suggest that effective VSI training is easily completed in less than 8 h. The thyroid VSI protocol can be performed within 10 min and is recommended to be performed with a highfrequency probe. There is no patient preparation required for the thyroid VSI examination which is shown in Fig. 2. Transverse sweeps of the midline, right, and left thyroid constitute the principal components of the thyroid VSI examination, while additional bilateral cine arcs of the probe are obtained in the longitudinal orientation for

\section{Thyroid Protocol}
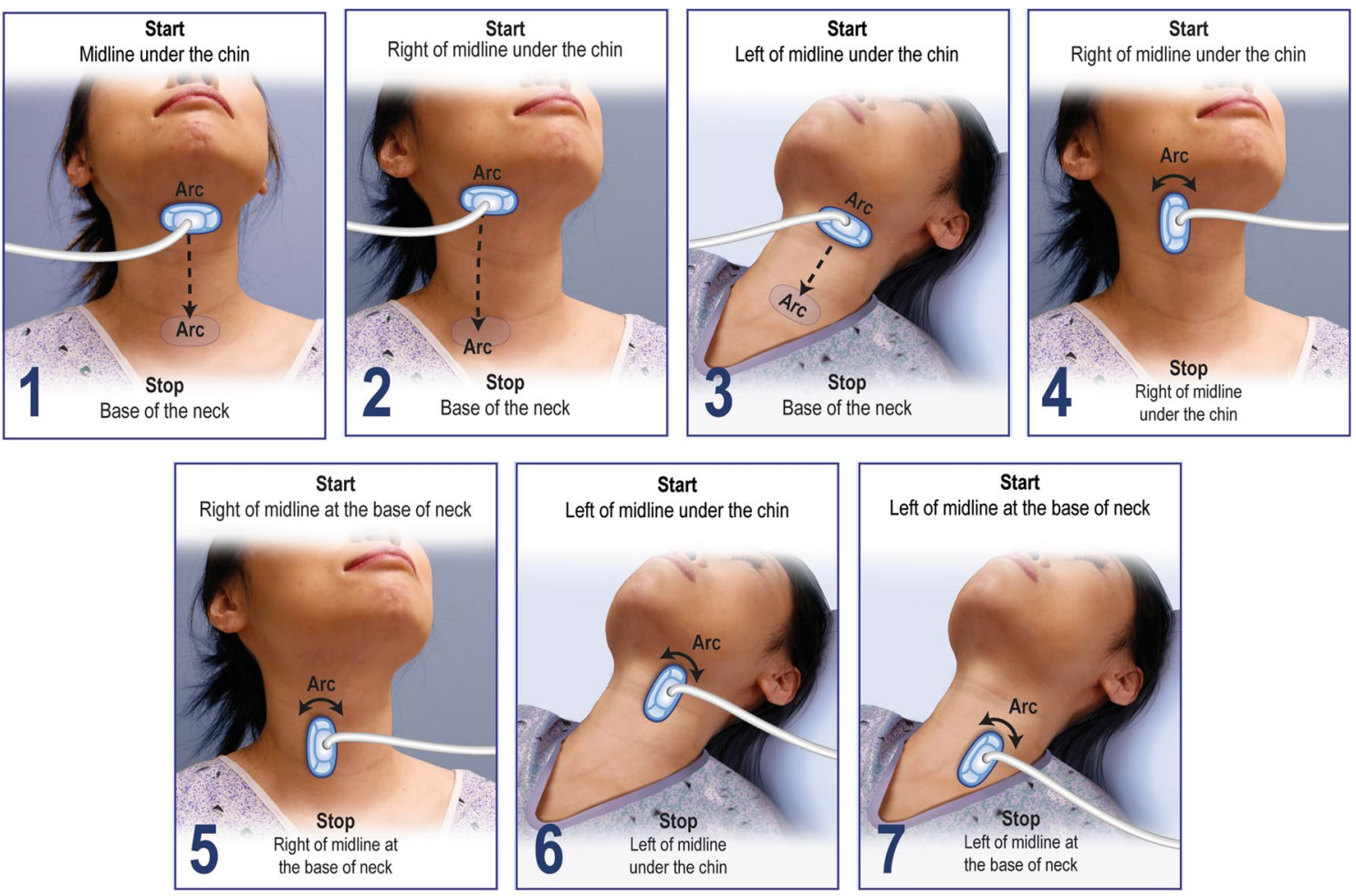

Fig. 2 Thyroid VSI protocol. The thyroid VSI protocol involves seven steps without patient preparation. The first three steps are transverse sweeps with an arc at the start and end of each sweep to maximize visibility. The last four sweeps are longitudinal arcs in the superior and inferior right and left thyroid to provide redundancy and localization information 
problem solving and redundancy. A video on how to perform the protocol and additional poster used in training are available as supplemental material (Supplemental Materials 1 and 2).

\section{Telemedicine system}

The "Medical for Ultrasound" or MED4US system was used for this study (Medical Innovation and Technology, Peru). This system has previously been described in detail with pilot testing showing reasonable send times at low bandwidths slightly faster than dialup internet [14]. The program is installed on a Windows 10 tablet which connects to the ultrasound machine. MED4US was designed to be used by rural health workers and is user-friendly, guiding the operator into obtaining the cine clips and entering relevant patient data to be sent to the interpreting physician. The tablet encrypts, compresses, and sends the cine clips to a cloud-based storage system for remote interpretation when internet is available. Remotely, a radiologist reviews the cine clips and completes a report which is sent back to the tablet to be given to the health center and patient.

\section{Image acquisition}

The Institutional Review Board at the Hospital Nacional Docente Madre Nino San Bartolome approved the study. Thyroid teleultrasound was piloted at the Conde de la Vega Health Center in Lima, Peru. The center serves primarily a low-income population and has a low to moderate $3 \mathrm{G}$ internet connection. Two ultrasound naïve trainees (a nurse and care technician) underwent didactic and hands-on training on the thyroid ultrasound protocol and telemedicine system over the course of $8 \mathrm{~h}$ in May 2018. Following training, both operators were certified to accurately perform the protocol by the training team. Subjects to be scanned were enrolled between June 2018 and March 2019 and were randomly recruited from patients over 18 years of age being seen at the clinic for various reasons. None of the study subjects were visiting the clinic for a primary thyroid related issue. The patient population served by the clinic is majority female.

The Mindray DP-10 ultrasound machine (Mindray, China) using standard thyroid settings with a $10 \mathrm{MHz}$ linear probe was employed for both standard of care and VSI examinations. After obtaining informed consent, the attending Peruvian radiologist performed the standard of care exam in accordance with standard ultrasound consensus guidelines and completed a report [3]. Next, the thyroid ultrasound VSI exam was performed, and the VSI exam cine clip sweeps were later sent for remote interpretation in the United States. No feedback on image quality was given to the VSI operators. The radiologist who performed and interpreted the standard of care exam informed patients of any clinically significant findings.

\section{Remote readings}

The VSI thyroid examinations were interpreted by two abdominal imaging attending radiologists with 7 and 40 years of experience, respectively. Each examination was scored on visualization of the isthmus and each thyroid lobe (less than $30 \%$ visualization, $30-50 \%$ visualization, $50-80 \%$ visualization, and greater than $80 \%$ visualization). Confidence in findings and image quality were also both scored using a three-point scale. Image quality was rated as poor, acceptable, or excellent. Excellent examinations visualized the entire or nearly entire thyroid gland with good brightness and resolution. Acceptable examinations visualized $>80 \%$ of the thyroid or had slightly limited brightness/resolution but still allowed for adequate gland or nodule assessment. Poor examinations visualized less than $80 \%$ of the total thyroid or were non-diagnostic. Confidence was scored as confident, intermediate confidence, or not confident. Both the anterior-posterior (AP) and transverse diameters of the thyroid lobes were measured. The length was not measured as the sagittal cine clips are split into upper and lower halves. Interpreters also estimated lobe size as small, normal, or large. Any nodule was measured in the largest dimension and characterized using free text with the readers commenting on the location, echogenicity, and presence of calcifications. In addition, the readers had the option of including free text comments with any image quality or study-related notes. Nodules less than $5 \mathrm{~mm}$ were not included in analysis as these are often clinically insignificant. After blinded readings, any discrepant cases between VSI and standard of care were examined to ascertain the source of disagreement.

\section{Statistical analysis}

Continuous variables were summarized by mean and standard deviation, and categorical variables were summarized by rate of occurrence and $95 \%$ confidence interval (CI). Agreement between VSI and standard of care ultrasound on the presence of nodules was quantified using Cohen's kappa. Agreement on lobe diameters was examined using intraclass correlation coefficients and Bland-Altman analysis. Intraclass correlation coefficients were calculated using a two-way random effects model for absolute agreement. Kappa values, intraclass correlation coefficients, and Bland-Altman biases were each compared to a theoretical mean of 0 using one-sample $t$ tests. Nodule size in the greatest dimension was compared 
with a paired $t$ test. All statistical analysis was performed using SPSS (v26, IBM Corporation, Armonk, NY) and MATLAB (R2019b, MathWorks, Natick, MA).

\section{Results}

Trainees scanned 121 subjects with the thyroid VSI protocol. The average age of subjects was $33.3 \pm 15$ years, and 98.3\% (94.2-99.8\%) of subjects were female. The blinded readers stated that longitudinal sweeps were useful in $67.8 \%$ $(58.7-76 \%)$ of cases for redundancy, nodule localization, and confirmation of findings. Sweeps were typically between 6 to $7 \mathrm{~s}$ resulting in less than one minute of cine clips for review. The raw file size for all clips combined was $6 \pm 1.46$ MB.

\section{Thyroid gland visualization and image quality}

The thyroid was well visualized in all exams with the vast majority visualizing the entire gland (Table 1 ). The left thyroid lobe and the isthmus showed $>80 \%$ visualization in all studies. The right thyroid lobe was slightly less well visualized with $88 \%$ of the studies showing $>80 \%$ visualization. The other $12 \%$ of exams showed $50-80 \%$ visualization of the right thyroid lobe. Furthermore, readers also noted seeing the majority of the thyroid on the first single midline transverse sweep highlighting the amenability of thyroid to the volume sweep imaging approach. There was only a single examination where the first midline transverse sweep did not completely image more than $80 \%$ of the left thyroid lobe. The right thyroid lobe was less optimally evaluated on

Table 1 Image quality and visualization

\begin{tabular}{lll}
\hline Measure & Level & $\begin{array}{l}\text { Percentage (95\% } \\
\text { confidence interval) }\end{array}$ \\
\hline Right lobe visualized & $<30 \%$ & - \\
& $30-50 \%$ & - \\
& $50-80 \%$ & $11.6 \%(6.47-18.7 \%)$ \\
& $\geq 80 \%$ & $87.6 \%(80.4-92.9 \%)$ \\
Left lobe visualized & $<30 \%$ & - \\
& $30-50 \%$ & - \\
& $50-80 \%$ & - \\
Isthmus visualized & $\geq 80 \%$ & $100 \%(97-100 \%)$ \\
& $<30 \%$ & - \\
& $30-50 \%$ & - \\
Image quality & $50-80 \%$ & - \\
& $\geq 80 \%$ & $100 \%(97-100 \%)$ \\
& Poor & - \\
& Acceptable & $12.4 \%(7.11-19.6 \%)$ \\
& Excellent & $87.6 \%(80.4-92.9 \%)$ \\
\hline
\end{tabular}

the first midline transverse sweep with $22 \%$ of the examinations showing $50-80 \%$ visualization (the other $78 \%$ showing $>80 \%$ visualization). The image quality was rated as excellent by blinded readers in $88 \%$ of cases with the thyroid well visualized with good image quality (Fig. 3 and Supplemental Material 3). Readers were confident in their estimations of lobe size and the presence of a nodule (Table 2).

\section{Thyroid measurements and nodule agreement}

Thyroid size measurements on VSI were, on average, within $5 \mathrm{~mm}$ of standard of care imaging (Table 3). There was fair to moderate agreement with intraclass correlation coefficients varying between 0.37 and 0.58 . The Bland-Altman bias varied between 2.84 and 1.07 , indicating that VSI tends to result in larger measurements compared to standard of
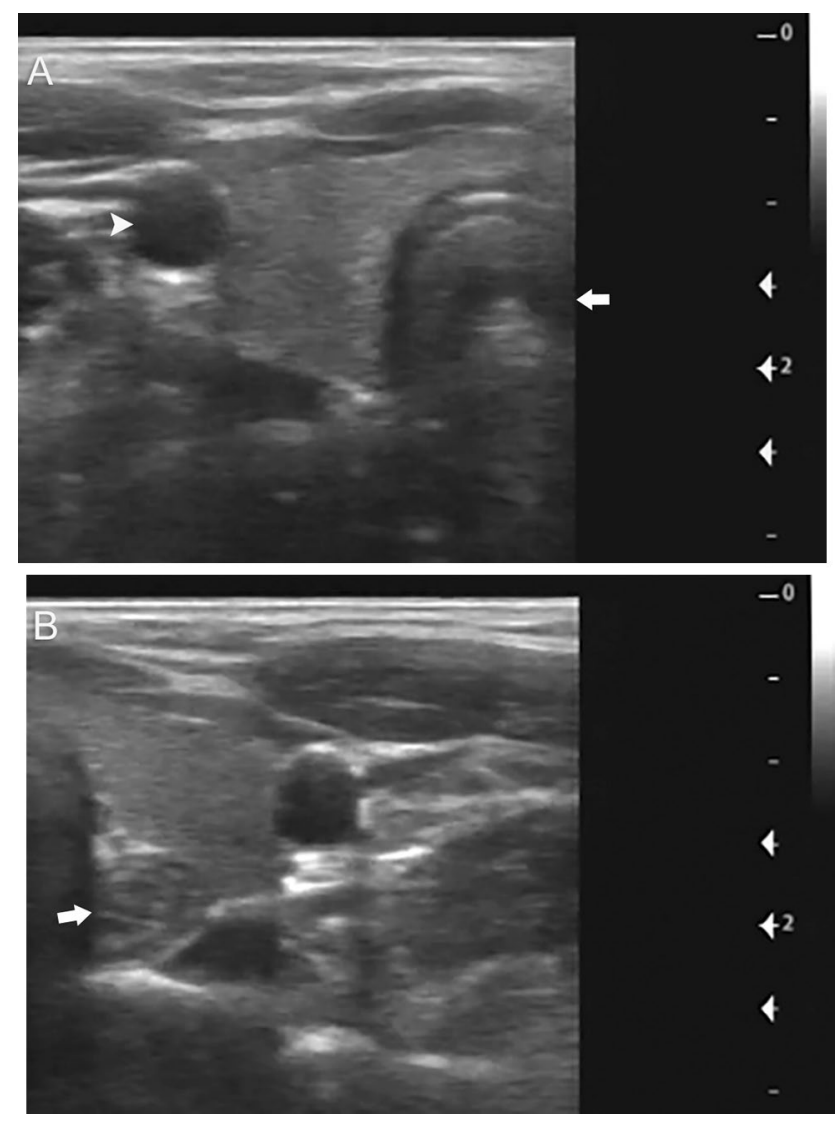

Fig. 3 Normal thyroid on VSI. Thyroid VSI examination from an asymptomatic 65 -year-old female performed by an operator after $8 \mathrm{~h}$ of training. a Normal right thyroid gland on a still image from the right transverse VSI cine clip. The trachea (arrow) and carotid artery (arrowhead) are also seen. b Normal left thyroid gland on a still image from the left transverse VSI cine clip. The esophagus is also seen (arrow). Supplemental Material 3 includes the cine clips from this exam. These cine clips and all other VSI examinations in this study were obtained by individuals without prior ultrasound experience 
Table 2 Lobe characteristics and confidence

\begin{tabular}{llll}
\hline Lobe & Variable & & Summary \\
\hline Right & Presence of nodule & & $4.13 \%(1.36-9.38 \%)$ \\
& Lobe size & Small & $2.48 \%(0.514-7.07 \%)$ \\
& & Normal & $95 \%(89.5-98.2 \%)$ \\
& Confidence & Not confident & $1.65 \%(0.201-5.84 \%)$ \\
& & Intermediate confidence & $0.826 \%(0.0209-4.52 \%)$ \\
& & Confident & $8.26 \%(4.03-14.7 \%)$ \\
Left & Presence of nodule & Small & $90.1 \%(83.3-94.8 \%)$ \\
& Lobe size & Normal & $4.96 \%(1.84-10.5 \%)$ \\
& & Large & $3.31 \%(0.908-8.25 \%)$ \\
& Confidence & Not confident & $95.9 \%(90.6-98.6 \%)$ \\
& & Intermediate confidence & $0.826 \%(0.0209-4.52 \%)$ \\
& & Confident & $0.826 \%(0.0209-4.52 \%)$ \\
& & & $4.13 \%(1.36-9.38 \%)$ \\
& Presence of nodule & Not confident & $95 \%(89.5-98.2 \%)$ \\
& Confidence & Intermediate confidence & $0.826 \%(0.0209-4.52 \%)$ \\
& & Confident & - \\
& & & $3.31 \%(0.908-8.25 \%)$ \\
& & $96.7 \%(91.8-99.1 \%)$ \\
\hline
\end{tabular}

Values are percentage (95\% confidence interval)

Table 3 Agreement on measurements

\begin{tabular}{llllll}
\hline Variable & \multicolumn{2}{l}{ Measurement $(\mathrm{mm})$} & & Agreement & \\
\cline { 2 - 3 } & VSI & Standard of care & & Intraclass correlation coefficient & Bland-Altman bias (VSI-standard of care) \\
\hline Right lobe AP & $16.4 \pm 2.8$ & $13.5 \pm 4.36$ & & $0.37(0.04-0.58, p=0.001)$ & $2.84(-5.71$ to $11.4, p<0.0001)$ \\
Right lobe transverse & $13.1 \pm 2.36$ & $12.1 \pm 2.24$ & & $0.57(0.35-0.71, p<0.0001)$ & $1.07(-3.69$ to $5.83, p<0.0001)$ \\
Left lobe AP & $14.5 \pm 2.83$ & $11.9 \pm 2.97$ & & $0.42(0.02-0.64, p<0.0001)$ & $2.54(-3.87$ to $8.94, p<0.0001)$ \\
Left lobe transverse & $12.9 \pm 2.32$ & $11.2 \pm 1.69$ & & $0.58(0.01-0.79, p<0.0001)$ & $1.77(-1.96$ to $5.5, p<0.0001)$ \\
Isthmus lobe AP & $3.73 \pm 0.858$ & $2.52 \pm 0.965$ & & $0.48(-0.22$ to $0.77, p<0.0001)$ & $1.21(-0.425$ to $2.84, p<0.0001)$ \\
\hline
\end{tabular}

Values are percentage (95\% confidence interval). Intraclass correlation coefficients were calculated using a two-way random effects model for absolute agreement

care imaging. Finally, there was excellent agreement on the presence of a nodule of $98.3 \%$ with a Cohen's kappa of 0.91 $(0.78-1, P<0.0001)$. VSI readers were also able to confidently and effectively characterize nodule characteristics including size, location, echogenicity, and the presence of calcifications in agreement with standard of care imaging (Fig. 4 and Supplemental Material 4).

There were 12 nodules identified on standard of care imaging - 4 solid, 4 mixed cystic and solid, and 4 cystic. Of these nodules, 4 were larger than $1 \mathrm{~cm}$. These studies with nodules represented $9.92 \%$ (5.23-16.7\%) of exams. The average nodule size in greatest dimension was $9.8 \mathrm{~mm} \pm 5.2$ $\mathrm{mm}$ (standard deviation) on VSI and $10.1 \mathrm{~mm} \pm 8 \mathrm{~mm}$ on standard of care imaging. There was no significant difference in nodule size $(P=0.74)$. Two nodules showed macrocalcifications which were both reported on standard of care imaging and VSI. All nodules were unknown to the patients being examined, but no follow-up information was subsequently obtained. There were only two examinations with discrepant nodule agreement between VSI and standard of care imaging. On consensus read, one examination was noted to be a missed nodule on VSI secondary to reader error. The other nodule discrepancy was not definitively resolved and may have represented a miss on standard of care imaging, but necessary standard of care images were not saved for definitive assessment. Finally, small nodules less than $5 \mathrm{~mm}$ were also detected on VSI and noted in the free text but not formally assessed as a part of this study. Additionally, a single goiter was identified in agreement between standard of care imaging and VSI. 

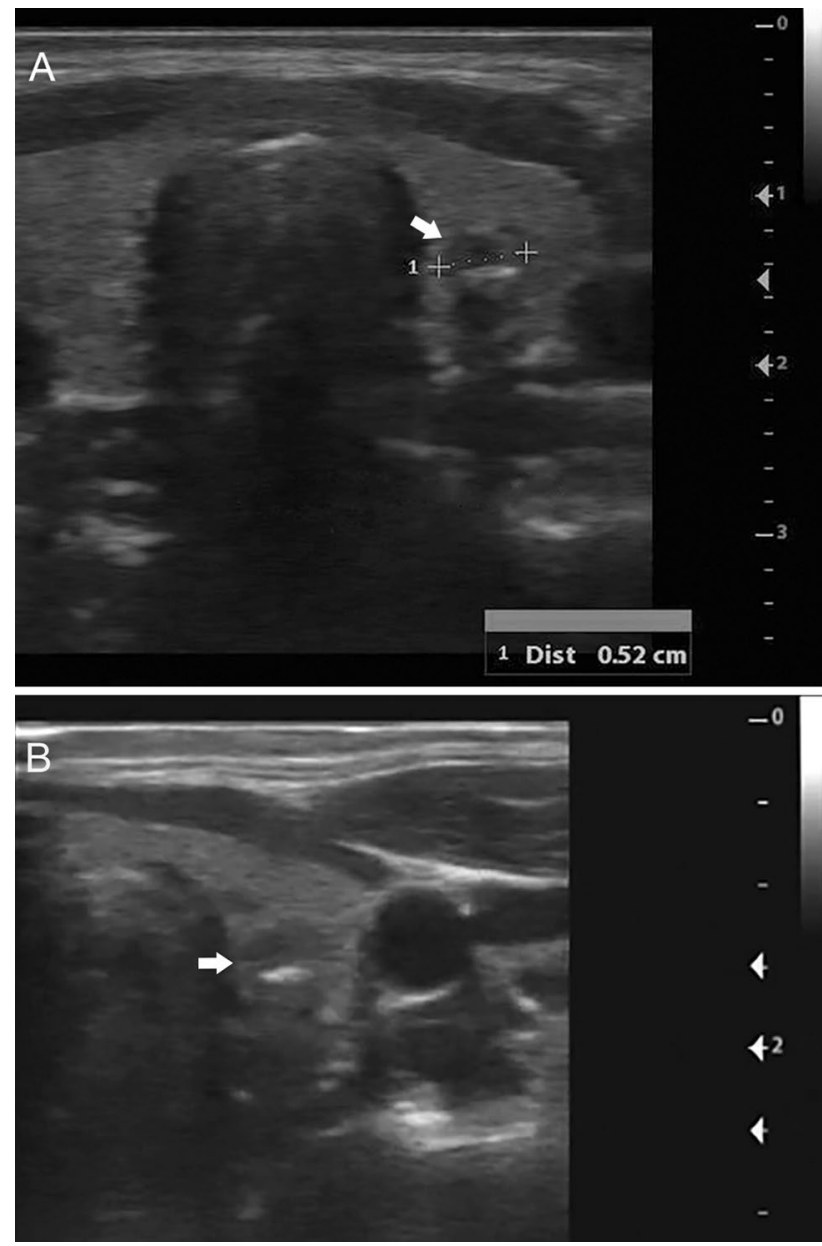

Fig. 4 Thyroid nodule on VSI. Thyroid VSI examination from an asymptomatic 31-year-old female. a Standard of care image showing a $5 \mathrm{~mm}$ solid hypoechoic thyroid nodule (arrow) in the middle third of the left thyroid with hyperechoic calcification. b Left transverse VSI sweep still image showing the same solid hypoechoic nodule (arrow) also measured at $5 \mathrm{~mm}$ with hyperechoic calcification. The left transverse VSI cine clip has been provided as Supplemental Material 4

\section{Discussion}

Correcting disparities in healthcare around the world requires a multifaceted approach, and improving access to medical imaging holds much promise in this regard. Thyroid ultrasound has an important role in the evaluation of thyroid nodules and goiter which are worldwide health issues. In this study, we have shown that after only a few hours of training, individuals without prior ultrasound experience were capable of obtaining images that fully visualized the thyroid and allowed identification and characterization of nodules. Furthermore, these novice operators consistently produced diagnostic examinations without ongoing feedback over the 9-month study duration. VSI measurements of the thyroid gland were, on average, within $5 \mathrm{~mm}$ of standard of care measurements, showing that VSI can accurately identify thyromegaly which may require further follow-up. From an epidemiological standpoint, we identified about a $10 \%$ prevalence of a thyroid abnormality in our asymptomatic sample, including a goiter. These findings are of indeterminate pathological significance but show the potential of this approach to be useful in future epidemiological studies. Given the advent of portable ultrasound machines, it is conceivable that widespread deployment of thyroid VSI teleultrasound could be realized with the goal of improving the health of the global community. In light of our findings, we propose several uses for this system: (1) evaluating a palpable thyroid nodule or gland, (2) following a known thyroid nodule, (3) assisting in epidemiological study of thyroid disease, and (4) assisting in thyroid education and awareness (iodine deficiency and its association with goiter).

The rationale behind the first three proposed uses has already been discussed in the introduction. In terms of improving thyroid education and awareness, there are many possibilities. Thyroid literacy has been predictably noted to be lower in underserved communities [41]. As an educational tool, thyroid ultrasound may assist clinicians in teaching their patients about topics such as iodine deficiency and its relation to goiter. Having a visual connection to a health problem may improve patient compliance and understanding of health issues [42-45]. There are additional potential ancillary benefits to adopting widespread use of thyroid VSI. For example, previous studies of VSI with obstetrics showed that more patients came to clinic when ultrasound was present, resulting in higher rates of prenatal testing [46]. Similar ancillary benefits could exist for thyroid VSI as more individuals may seek care, resulting in improved health, education, and public awareness of thyroid disease.

The ultrasound novices in this study performed well acquiring examinations of universally acceptable to excellent image quality. While we did not notice a decrease in image quality over the study period, the use of refresher courses on VSI could also be considered as a way to ensure continued quality. Even with excellent image quality in the majority of examinations, it is possible some small nodules may be better served by dedicated sonographic evaluation with highresolution imaging by an expert. Similarly, increased body habitus or otherwise abnormal neck anatomy may limit the VSI protocol's ability to assess the thyroid. Future studies could assess the protocol's performance in these circumstances. In addition, the number of nodules larger than $1 \mathrm{~cm}$ in this sample was relatively small. Future studies might be conducted in thyroid clinics with a sample of more disease or in populations with goiter. Length measurements of the thyroid are currently not obtainable due to the split nature of the longitudinal clips. This could be potentially solved with advanced imaging processing or revised future iterations of 
the protocol. Artificial intelligence could also allow for identification of nodules and thyroid size at the time of study.

Although there are characteristic features of hyperthyroidism and hypothyroidism on ultrasound, imaging is not recommended in the routine evaluation of these conditions [46 48, 49]. As with standard of care thyroid ultrasound, there exists the theoretical risk for over-screening of thyroid nodules [50-52]. This issue may be mitigated by the increasing use of the Thyroid Imaging Reporting and Data System (TI-RADS) which improves accuracy and may reduce the number of biopsies prompted by ultrasound [53]. VSI can adequately assess for the features of TIRADS allowing for standardized management of findings. Evidence also suggests that it is important to note the clinical context of each individual patient when considering biopsy [54]. Our telediagnostic system also provides this relevant clinical information to the interpreting provider. At this time, we are not advocating this protocol be used to screen asymptomatic people outside of epidemiological studies. Some clinicians may be concerned regarding legal exposure from use of VSI examinations. In general, the alternative to clinical use of thyroid VSI in many rural and underserved areas would be no imaging at all. Given the potential clinical benefits in this context, significant legal exposure is not anticipated. Furthermore, review of the legal literature shows no lawsuits related to point of care ultrasound use, and, in fact, lawsuits were identified when ultrasound was failed to be performed [55, 56].

Thyroid disease is a prevalent health issue worldwide with a majority of the world lacking access to diagnostic imaging for its evaluation [2]. The examinations in this study obtained by individuals without prior ultrasound experience showed excellent agreement with standard of care imaging for thyroid size and nodule evaluation. As rates of thyroid cancer continue to increase, thyroid VSI telediagnostic ultrasound could be a cost-effective asset both in determining clinical management and in conducting epidemiological study. Deployment of this system is feasible with significant potential to improve medical care of those in need, especially in rural and underserved areas.

Supplementary Information The online version contains supplementary material available at https://doi.org/10.1007/s40618-021-01584-7.

Acknowledgements We thank Medical Imaging Ministries of the Americas and Medical Innovation and Technology for allowing the publication of their illustrated poster and video. We thank Dr. Deborah Rubens, Dr. Michael Potchen, and Dr. Gretchen Birbeck for their mentorship, global health knowledge, and thyroid ultrasound expertise. We thank Nadezhda Kiriyak, Sarah Klingenberger, Jane Lichorowic, and Gwen Mack for their assistance with the figures and illustrations.

Author contributions All listed authors contributed to the design, data acquisition, data analysis, data interpretation, and/or drafting of the final manuscript. All authors approved the final version of the manuscript submitted.

Funding Authors acknowledge the following funding sources: Innóvate Peru (409-FIDECOM INNOVATEPERU-PVE-2017), Pontifical Catholic University of Peru (Período de Investigación 2020-2021).

Availability of data and material The data sets generated in this study are not publically available as an additional measure to protect subject privacy. However, all relevant data have been presented in this article. Please address any inquiries regarding data use to Dr. Castaneda.

Code availability Not applicable.

\section{Declarations}

Conflict of interest Benjamin Castaneda and all authors affiliated with Medical Innovation and Technology have financial stake in Medical Innovation and Technology which works on developing technology to bring medical imaging to rural areas. All other authors have no financial disclosures. The manuscript and its contents were produced by the University of Rochester and Medical Imaging Ministries of the Americas authors without financial conflict. The Medical Innovation and Technology authors previewed the document, and any changes were subject to approval from the University of Rochester and Medical Imaging Ministry of the Americas authors.

Ethical approval This study was conducted in accordance with the 1964 Helsinki Declaration and was approved by the Institutional Review Board at the Hospital Nacional Docente Madre Nino San Bartolome.

Informed consent All individuals who participated in this study provided informed consent.

Consent for publication All individuals who participated in this study provided consent to publish.

Open Access This article is licensed under a Creative Commons Attribution 4.0 International License, which permits use, sharing, adaptation, distribution and reproduction in any medium or format, as long as you give appropriate credit to the original author(s) and the source, provide a link to the Creative Commons licence, and indicate if changes were made. The images or other third party material in this article are included in the article's Creative Commons licence, unless indicated otherwise in a credit line to the material. If material is not included in the article's Creative Commons licence and your intended use is not permitted by statutory regulation or exceeds the permitted use, you will need to obtain permission directly from the copyright holder. To view a copy of this licence, visit http://creativecommons.org/licenses/by/4.0/.

\section{References}

1. The Lancet D, Endocrinology (2013) The untapped potential of the thyroid axis. Lancet Diabetes Endocrinol 1(3):163. https://doi. org/10.1016/S2213-8587(13)70166-9

2. World Radiography Day: Two-Thirds of the World's Population has no Access to Diagnostic Imaging. Pan American Health Organization. https://www.paho.org/hq/index.php?option=com content $\&$ view $=$ article $\&$ id $=7410: 2012$-dia-radiografia-dos-terci os-poblacion-mundial-no-tiene-acceso-diagnostico-imagen \& 
Itemid=1926\&lang=en\#: : text=\%2D $\% 20$ The $\% 20$ use $\% 20$ of $\%$ 20X\%2Drays,no $\% 20$ access $\% 20$ to $\% 20$ diagnostic $\% 20$ imaging.\& text $=$ It $\% 20$ is $\% 20$ used $\% 20$ for $\% 20$ diagnostic $\% 2 \mathrm{C} \% 20$ preventive $\%$ 2C\%20and\%20therapeutic\%20purposes. Accessed 30 Oct 2020

3. (2013)AIUM practice guideline for the performance of a thyroid and parathyroid ultrasound examination. J Ultrasound Med 32(7):1319-1329. https://doi.org/10.7863/ultra.32.7.1319

4. Chaudhary V, Bano S (2013) Thyroid ultrasound. Indian J Endocrinol Metab 17(2):219-227. https://doi.org/10.4103/2230-8210. 109667

5. Maru DS, Schwarz R, Jason A, Basu S, Sharma A, Moore C (2010) Turning a blind eye: the mobilization of radiology services in resource-poor regions. Glob Health 6:18. https://doi.org/ 10.1186/1744-8603-6-18

6. Mollura DJ, Lungren MP (2014) Radiology in global health: strategies, implementation, and applications. Springer, New York

7. Mollura DJ, Mazal J, Everton KL, Group R-ACW (2013) White paper report of the 2012 RAD-AID conference on International Radiology for Developing Countries: planning the implementation of global radiology. J Am Coll Radiol 10(8):618-624. https://doi. org/10.1016/j.jacr.2013.01.019

8. Ngoya PS, Muhogora WE, Pitcher RD (2016) Defining the diagnostic divide: an analysis of registered radiological equipment resources in a low-income African country. Pan Afr Med J 25:99. https://doi.org/10.11604/pamj.2016.25.99.9736

9. Britton N, Miller MA, Safadi S, Siegel A, Levine AR, McCurdy MT (2019) Tele-ultrasound in resource-limited settings: a systematic review. Front Public Health 7:244. https://doi.org/10.3389/ fpubh.2019.00244

10. Kim T, Zuckerman JE (2019) Realizing the potential of telemedicine in global health. J Glob Health 9(2):020307. https://doi.org/ 10.7189/jogh.09.020307

11. Popov V, Popov D, Kacar I, Harris RD (2007) The feasibility of real-time transmission of sonographic images from a remote location over low-bandwidth Internet links: a pilot study. AJR Am J Roentgenol 188(3):W219-222. https://doi.org/10.2214/AJR.05. 2148

12. Scott Kruse C, Karem P, Shifflett K, Vegi L, Ravi K, Brooks M (2018) Evaluating barriers to adopting telemedicine worldwide: a systematic review. J Telemed Telecare 24(1):4-12. https://doi. org/10.1177/1357633X16674087

13. Marini TJ, Oppenheimer DC, Baran TM, Rubens DJ, Toscano M, Drennan K, Garra B, Miele FR, Garra G, Noone SJ, Tamayo L, Carlotto C, Trujillo L, Waks E, Garra K, Egoavil MS, Berrospi J, Castaneda B (2020) New ultrasound telediagnostic system for low-resource areas: pilot results from Peru. J Ultrasound Med. https://doi.org/10.1002/jum.15420

14. Ferrer J, Chaumont T, Trujillo L, Fernandez I, Guerrero J, Stewart P, Garra G, Campos MF, Garra K, Stephens N, Harley C, Jacobo S, Waks E, Miele F, Garra B, Castaneda B (2017) New tele-diagnostic model using volume sweep imaging for rural areas. Conf Proc IEEE Eng Med Biol Soc 2017:2622-2625. https://doi. org/10.1109/EMBC.2017.8037395

15. Health Care Broadband in America (2010) Federal Communications Commission. https://www.fcc.gov/document/obi-technicalpaper-no-5-health-care-broadband-america

16. Measuring Digital Development Facts and Figures (2019) International Telecommunication Union. https://www.itu.int/myitu/-/ media/Publications/2020-Publications/Measuring-digital-devel opment-2019.pdf

17. Mesele M, Degu G, Gebrehiwot H (2014) Prevalence and associated factors of goiter among rural children aged 6-12 years old in Northwest Ethiopia, cross-sectional study. BMC Public Health 14:130. https://doi.org/10.1186/1471-2458-14-130

18. Pretell EA, Moncloa F, Salinas R, Kawano A, Guerra-Garcia R, Gutierrez L, Beteta L, Pretell J, Wan M (1969) Prophylaxis and treatment of endemic goiter in Peru with iodized oil. J Clin Endocrinol Metab 29(12):1586-1595. https://doi.org/10.1210/ jcem-29-12-1586

19. Stanbury JB, Kevany JP (1970) Iodine and thyroid disease in Latin America. Environ Res 3(4):353-363. https://doi.org/10.1016/ 0013-9351(70)90028-9

20. Unnikrishnan AG, Menon UV (2011) Thyroid disorders in India: an epidemiological perspective. Indian J Endocrinol Metab 15(Suppl 2):S78-81. https://doi.org/10.4103/2230-8210.83329

21. Abraham D, Singh N, Lang B, Chan WF, Lo CY (2007) Benign nodular goitre presenting as acute airway obstruction. ANZ J Surg 77(5):364-367. https://doi.org/10.1111/j.1445-2197.2007.04061.x

22. Başoğlu M, Öztürk G, Aydınlı B, Yıldırgan M, Atamanalp SS, Celebi F (2009) Benign nodular goiter causing upper airway obstruction. Eurasian J Med 41(2):75-79

23. Sajid B, Rekha K (2017) Airway management in patients with tracheal compression undergoing thyroidectomy: a retrospective analysis. Anesth Essays Res 11(1):110-116. https://doi.org/10. 4103/0259-1162.186608

24. Pang HN, Chen CM (2007) Incidence of cancer in nodular goitres. Ann Acad Med Singap 36(4):241-243

25. Smith JJ, Chen X, Schneider DF, Broome JT, Sippel RS, Chen H, Solórzano CC (2013) Cancer after thyroidectomy: a multiinstitutional experience with 1,523 patients. J Am Coll Surgeons 216(4):571-577. https://doi.org/10.1016/j.jamcollsurg.2012.12. 022 (discussion 577-579)

26. Borges AKdM, Miranda-Filho A, Koifman S, Koifman RJ (2017) Thyroid cancer incidences from selected south america population-based cancer registries: an age-period-cohort study. J Glob Oncol 4:1-11. https://doi.org/10.1200/JGO.17.00024

27. Deng Y, Li H, Wang M, Li N, Tian T, Wu Y, Xu P, Yang S, Zhai Z, Zhou L, Hao Q, Song D, Jin T, Lyu J, Dai Z (2020) Global burden of thyroid cancer from 1990 to 2017. JAMA Netw Open 3(6):e208759. https://doi.org/10.1001/jamanetworkopen.2020. 8759

28. Kitahara CM, Sosa JA (2016) The changing incidence of thyroid cancer. Nat Rev Endocrinol 12(11):646-653. https://doi.org/10. 1038/nrendo.2016.110

29. López Gavilanez E, Bautista Litardo N, Navarro Chávez M, Hernández Bonilla M, Segale Bajaña A (2020) Thyroid cancer in Ecuador. BMC Cancer 20(1):637. https://doi.org/10.1186/ s12885-020-07137-0

30. Pellegriti G, Frasca F, Regalbuto C, Squatrito S, Vigneri R (2013) Worldwide increasing incidence of thyroid cancer: update on epidemiology and risk factors. J Cancer Epidemiol 2013:965212. https://doi.org/10.1155/2013/965212

31. Salazar-Vega J, Ortiz-Prado E, Solis-Pazmino P, Gómez-Barreno L, Simbaña-Rivera K, Henriquez-Trujillo AR, Brito JP, Toulkeridis T, Coral-Almeida M (2019) Thyroid cancer in Ecuador, a 16 years population-based analysis (2001-2016). BMC Cancer 19(1):294. https://doi.org/10.1186/s12885-019-5485-8

32. Seib CD, Sosa JA (2019) Evolving understanding of the epidemiology of thyroid cancer. Endocrinol Metab Clin N Am 48(1):23-35. https://doi.org/10.1016/j.ecl.2018.10.002

33. Sierra MS, Soerjomataram I, Forman D (2016) Thyroid cancer burden in Central and South America. Cancer Epidemiol 44(Suppl 1):S150-s157. https://doi.org/10.1016/j.canep.2016. 07.017

34. Marqusee E, Benson CB, Frates MC, Doubilet PM, Larsen PR, Cibas ES, Mandel SJ (2000) Usefulness of ultrasonography in the management of nodular thyroid disease. Ann Intern Med 133(9):696-700. https://doi.org/10.7326/0003-4819-133-9-20001 1070-00011

35. Tollin SR, Mery GM, Jelveh N, Fallon EF, Mikhail M, Blumenfeld W, Perlmutter S (2000) The use of fine-needle aspiration biopsy under ultrasound guidance to assess the risk of malignancy in 
patients with a multinodular goiter. Thyroid 10(3):235-241. https://doi.org/10.1089/thy.2000.10.235

36. Salazar-Vega J, Ortiz-Prado E, Solis-Pazmino P, Gomez-Barreno L, Simbana-Rivera K, Henriquez-Trujillo AR, Brito JP, Toulkeridis T, Coral-Almeida M (2019) Thyroid cancer in Ecuador, a 16 years population-based analysis (2001-2016). BMC Cancer 19(1):294. https://doi.org/10.1186/s12885-019-5485-8

37. DeStigter K, Morey G, Garra B, Rielly M, Anderson M, Kawooya MG, Matovu A, Miele F Low-cost teleradiology for rural ultrasound. In: Proceedings of the IEEE global humanitarian technology conference (GHTC). Seattle, Washington, Oct 30-Nov 1, 2011. IEEE Press, pp 290-295

38. Toscano M, Marini TJ, Drennan K et al (2021) Testing telediagnostic obstetric ultrasound in Peru: a new horizon in expanding access to prenatal ultrasound. BMC Pregnancy Childbirth 21:328(2021). https://doi.org/10.1186/s12884-021-03720-w

39. Dougherty A, Kasten M, DeSarno M, Badger G, Streeter M, Jones DC, Sussman B, DeStigter K (2020) Validation of a telemedicine quality assurance method for point-of-care obstetric ultrasound used in low-resource settings. J Ultrasound Med. https://doi.org/ 10.1002/jum. 15429

40. Marini T, Castaneda B, Baran T, O'Connor T, Garra B, Tamayo L, Zambrano M, Carlotto C, Trujillo L, Kaproth-Joslin K (2019) Lung ultrasound volume sweep imaging for pneumonia detection in rural areas: piloting training in rural Peru. J Clin Imaging Sci 9(35). https://clinicalimagingscience.org/lung-ultrasound-volumesweep-imaging-for-pneumonia-detection-in-rural-areas-pilotingtraining-in-rural-peru/

41. Perumal SS, Prasad S, Surapaneni KM, Joshi A (2015) Health information-seeking behavior among hypothyroid patients at Saveetha Medical College and Hospital. Ethiop J Health Sci 25(2):147-154. https://doi.org/10.4314/ejhs.v25i2.7

42. Alamantariotou K, Zisi D (2010) Consumer health informatics and interactive visual learning tools for health. Int J Electron Healthc 5(4):414-424. https://doi.org/10.1504/ijeh.2010.036211

43. Beacom AM, Newman SJ (2010) Communicating health information to disadvantaged populations. Fam Community Health 33(2):152-162. https://doi.org/10.1097/FCH.0b013e3181d59344

44. Entwistle V, Williams B (2008) Health literacy: the need to consider images as well as words. Health Expect Int J Public Particip Health Care Health Policy 11(2):99-101. https://doi.org/10. 1111/j.1369-7625.2008.00509.x

45. Peregrin T (2010) Picture this: visual cues enhance health education messages for people with low literacy skills. J Am Diet Assoc 110(4):500-505. https://doi.org/10.1016/j.jada.2010.02.019

46. Ross AB, DeStigter KK, Rielly M, Souza S, Morey GE, Nelson M, Silfen EZ, Garra B, Matovu A, Kawooya MG (2013) A low-cost ultrasound program leads to increased antenatal clinic visits and attended deliveries at a health care clinic in rural Uganda. PLoS ONE 8(10):e78450. https://doi.org/10.1371/journal.pone.00784 50

47. Ceylan I, Yener S, Bayraktar F, Secil M (2014) Roles of ultrasound and power Doppler ultrasound for diagnosis of Hashimoto thyroiditis in anti-thyroid marker-positive euthyroid subjects. Quant Imaging Med Surg 4(4):232-238

48. Pedersen OM, Aardal NP, Larssen TB, Varhaug JE, Myking O, Vik-Mo H (2000) The value of ultrasonography in predicting autoimmune thyroid disease. Thyroid 10(3):251-259. https://doi.org/ 10.1089/thy.2000.10.251

49. Trimboli P, Rossi F, Condorelli E, Laurenti O, Ventura C, Nigri G, Romanelli F, Guarino M, Valabrega S (2010) Does normal thyroid gland by ultrasonography match with normal serum thyroid hormones and negative thyroid antibodies? Exp Clin Endocrinol Diabetes 118(9):630-632. https://doi.org/10.1055/s-0029-12377 00

50. Morris LG, Sikora AG, Tosteson TD, Davies L (2013) The increasing incidence of thyroid cancer: the influence of access to care. Thyroid 23(7):885-891. https://doi.org/10.1089/thy.2013. 0045

51. Sanabria A, Kowalski LP, Shah JP, Nixon IJ, Angelos P, Williams MD, Rinaldo A, Ferlito A (2018) Growing incidence of thyroid carcinoma in recent years: factors underlying overdiagnosis. Head Neck 40(4):855-866. https://doi.org/10.1002/hed.25029

52. Vigneri R, Malandrino P, Vigneri P (2015) The changing epidemiology of thyroid cancer: why is incidence increasing? Curr Opin Oncol 27(1):1-7. https://doi.org/10.1097/cco.0000000000000148

53. Grant EG, Tessler FN, Hoang JK, Langer JE, Beland MD, Berland LL, Cronan JJ, Desser TS, Frates MC, Hamper UM, Middleton WD, Reading CC, Scoutt LM, Stavros AT, Teefey SA (2015) Thyroid ultrasound reporting lexicon: white paper of the ACR Thyroid Imaging, Reporting and Data System (TIRADS) Committee. J Am Coll Radiol 12(12 Pt A):1272-1279. https://doi.org/10.1016/j.jacr. 2015.07.011

54. Magri F, Chytiris S, Croce L, Molteni M, Bendotti G, Gruosso G, Tata Ngnitejeu S, Agozzino M, Rotondi M, Chiovato L (2020) Performance of the ACR TI-RADS and EU TI-RADS scoring systems in the diagnostic work-up of thyroid nodules in a real-life series using histology as reference standard. Eur J Endocrinol 183(5):521-528. https://doi.org/10.1530/eje-20-0682

55. Blaivas M, Pawl R (2012) Analysis of lawsuits filed against emergency physicians for point-of-care emergency ultrasound examination performance and interpretation over a 20 -year period. Am J Emerg Med 30(2):338-341. https://doi.org/10.1016/j.ajem.2010. 12.016

56. Stolz L, O’Brien KM, Miller ML, Winters-Brown ND, Blaivas M, Adhikari S (2015) A review of lawsuits related to point-of-care emergency ultrasound applications. West J Emerg Med 16(1):1-4. https://doi.org/10.5811/westjem.2014.11.23592

Publisher's Note Springer Nature remains neutral with regard to jurisdictional claims in published maps and institutional affiliations. 